\section{ANNALS OF THE UNIVERSITY OF CRAIOVA}

\author{
Series: $\checkmark$ Biology \\ $\checkmark$ Horticulture \\ $\checkmark$ Food products processing \\ technology \\ $\checkmark$ Environmental engineering
}

\title{
Vol. XXVI (LXII) - 2021 \\ CHARACTERIZATION AND EVALUATION OF SOME POTATO CLONES REGARDING THE BEHAVIOR TO HYDRIC STRESS IN VITRO CONDITIONS
}

\author{
Hermeziu Manuela ${ }^{1}$, Tican Andreea ${ }^{1}$, Hermeziu Radu${ }^{1}$, Chelmea Carmen ${ }^{1}$ \\ ${ }^{1}$ National Institute of Research and Development for Potato and Sugar Beet Brasov (Romania) \\ ${ }^{*}$ Corresponding author, e-mail: hermezium@gmail.com
}

Keywords: potato, culture medium, in vitro, hydric stress

\begin{abstract}
Potato (Solanum tuberosum L.), the third (after wheat, corn and rice), most important food in the world is currently suffering from climate change. The potato crop is strongly influenced by the amount of precipitation and their distribution. The present study was conducted to determine the cultural behavior, respectively the response to hydric stress of some clones from the National Institute of Research and Development for Potato and Sugar Beet Brasov. Two factors were tested: factor I - potato breeding lines 27; factor II - culture medium (clasic medium Murashige- Skoog and medium Murashige- Skoog with osmotic agent). 1982/3 clone was identified with high values and significant differences in case of medium with PEG in $2 \%$ concentration, for the number of leaves/plantlets and for root lenght. Also, 1939/2 clone was identified with high values for the number of leaves/plantlets and 1982/1 clone for the root length. 1958/3 clone presented high values for two of the analyzed parameters: plantlet length and fresh plantlets weight, when was applied PEG $2 \%$ in the nutrition medium.
\end{abstract}

\section{INTRODUCTION}

Potato regularly suffers from water scarcity in most regions where it is grown due to irregular rainfall or inadequate irrigation techniques. Potato crop often needs additional irrigation, which is an unavailable resource in many locations. (Obidiegwu 2015). Changing climatic conditions that lead to higher temperatures, dry periods, will increasingly affect potato production in many parts of the world (Evers et al, 2010).

Improving drought tolerance of potatoes through conventional breeding methods or biotechnology should be promoted. The aim is to considerably reduce the time of creation of a new variety, so that at the time of its identification there is already biological material with the desired characteristics, to be used for the propagation process (Cabello 2013).

At high temperatures, in drought conditions, the temperature of the leaf rises well above the air temperature. Thus, the stomata close, the perspiration process is stopped, the photosynthesis process ceases and the leaf withers (Monneveux, 2014). High soil temperatures are more harmful to potato production than high air temperatures.

Drought impairs mitosis, cell elongation and expansion,resulting in a reduction in plant length, leaf area and crop growth (Hussain et al., 2008, Albiski, 2012).

Reduction of leaf size is the first morphological manifestation due to drought (Jefferies and MacKerron, 1987) associated with reduced light interception 
and leads to a reduction in dry matter accumulation (Deblonde et al., 1999 ). Drought conditions also affect nutrient transport to the root surface by inducing root shrink-age and subsequent loss of the soil-root contact (Ahmad et al., 2013). Water scarcity, which often appears in our regions with a temperate continental climate, restricts plant growth or forces early maturation in many cases, especially when it is associated with temperatures above the threshold level (Morar, 1999). Considering the influence of climate change and the fact that the potato crop has reduced genetic variability it is necessary to identify drought tolerant or droughtresistant genetic material (Baciu 2013).

In vitro conditions are a very controlled and homogeneous procedure to rapidly induce osmotic stress (Manoj and Uday, 2007). Polyethylene glycol (PEG) is widely used in vitro to simulate water stress. It is a non-penetrating inert osmotic (high molecular weight) compound that reduces the potential of nutrient solutions without being taken up by the plant or showing phytotoxicity (Hassan et al., 2004). With the addition of polyethylene glycol to the culture medium, it becomes difficult to absorb seedling water from the nutrient medium and thus simulates the effect of drought on the microplantula.

In vitro osmotic stress, induced by polyethylene glycol, has been used to evaluate root and shoot traits at the juvenile stage and for recovery potential (Gopal et al., 2008). Polyethylene glycol of large molecular weight is taken up slower by plants plant, thus representing a better osmoticum for use in hydroponics root medium (Yaniv et al., 1983). Mannitol solution is also used for inducing drought stress in tissue culture. It is however taken up into plant tissues in maize, wheat, rape and potato (Lipavska and Vreugdenhil, 1996), causing artifact effects (Monneveux et al., 2013).

\section{MATERIAL AND METHODS}

In the Research Laboratory for Plant Tissue Cultures of NIRDPSB Brasov was used a method of selection for some potato clones.

To determine the resistance to in vitro water stress conditions was performed a bifactorial experiment $(27 \times 2)$ with 3 repetitions, including the following factors:

- Experimental factor A - potato genotype, with 27 graduations. For this factor we considered as control, the mean of the values obtained for each parameter studied.

- Experimental factor B - culture medium used with two graduations:

- $b_{1}$ - MS control medium, to which no osmotic agent was added;

- $b_{2}$ - MS medium, to which $2 \%$ PEG (polyethylene glycol) was added.

Initially a thermic regime of $18^{\circ} \mathrm{C}$ was ensured, the tubers being kept in the light conditions until the shoots reached $2-3 \mathrm{~cm}$ in length.

The tuber shoots were then sterilized (for 10-15 minutes, depending on the size of the shoots) using a solution based on sodium hypochlorite $(\mathrm{NaOCl})$, then washed three times with sterilized distilled water; sterilize with refined alcohol $96^{\circ}$ for three minutes; wash again three times with sterilized distilled water and dry on sterilized paper in the laminar flow hood). The shoots were inoculated into the culture medium, the test tubes were transferred to the growth chamber, under controlled conditions of temperature and light, where after 3-4 weeks the plantlets developed. The plantlets obtained were multiplied at the level of each internode, and the mini-cuttings obtained were inoculated on two types of medium: the classical 
medium Murashige - Skoog (1962) (MS) and the medium for inducing water stress, by adding $2 \%$ PEG, in the classical medium.

After 4 weeks were made the following determinations: plantlets length, number of leaves / plantlets, root length, fresh plantlets weight and fresh root weight.

The results were processed by the analysis of variation and the significance of the differences was established by the method of multiple comparisons.

\section{RESULTS AND DISSCUSSIONS}

PEG has a high molecular (6000) weight and is used in inducing drought in vitro, as a water-lowering agent in a manner similar to soil drying. The addition of PEG to the culture medium results in a reduction in the number of leaves, plantlets length and root length. PEG acted as an osmotic agent resulting in reduced root water and nutrients absorption.

Regarding the length of the plantlets, the clone 1958/3 was noted with very significant positive difference $(8.22 \mathrm{~cm})$ for the culture medium that contained PEG $2 \%$. Also, 1982/2A clone had a distinctly significant positive difference $(6.39 \mathrm{~cm})$ and $1962 / 3$ clone had a positive significant difference $(4.05 \mathrm{~cm})$, both on culture medium with PEG 2\% (Table 1). These clones can produce plants with stems capable of growth and development under drought conditions.

Table 1

Nutrition medium and clones combined influence on plantlets length $(\mathrm{cm})$

\begin{tabular}{|c|c|c|c|c|c|c|c|c|}
\hline \multirow{2}{*}{$\begin{array}{c}\text { Clone (a)/ } \\
\text { Culture } \\
\text { medium (b) }\end{array}$} & \multicolumn{8}{|c|}{ Plantlets lenght $(\mathrm{cm})$} \\
\hline & $\begin{array}{l}\text { Medium } \\
\left.\text { MS (b } b_{1}\right)\end{array}$ & $\begin{array}{l}\text { Diff. } \\
\text { (cm) }\end{array}$ & Sign. & $\begin{array}{c}\text { Medium } \\
\text { MS+PEG2\% (b } 2)\end{array}$ & $\begin{array}{l}\text { Diff. } \\
\text { (cm) }\end{array}$ & Sign. & $\mathbf{b}_{2}-\mathbf{b}_{1}$ & Sign. \\
\hline $1939 / 4$ & 1,50 & $-5,39$ & 00 & 2,00 & $-1,95$ & ns & 0,50 & ns \\
\hline $1962 / 1$ & 3,23 & 3,65 & ns & 4,27 & 0,32 & ns & 1,03 & ns \\
\hline $1962 / 2$ & 4,67 & $-2,22$ & ns & 4,33 & 0,39 & ns & $-0,33$ & ns \\
\hline $1941 / 11$ & 4,20 & $-2,69$ & ns & 0,80 & $-3,15$ & ns & $-3,40$ & ns \\
\hline $1958 / 3$ & 12,67 & 5,78 & ** & 12,17 & 8,22 & 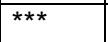 & $-0,50$ & ns \\
\hline $1941 / 15$ & 1,77 & $-5,12$ & 00 & 4,17 & 0,22 & ns & 2,40 & ns \\
\hline $1976 / 1$ & 6,33 & $-0,55$ & ns & 2,40 & $-1,55$ & ns & $-3,93$ & 0 \\
\hline $1939 / 2$ & 6,73 & 0,15 & ns & 4,40 & 0,45 & ns & $-2,33$ & ns \\
\hline $1901 / 12$ & 6,67 & $-0,22$ & ns & 2,50 & $-1,45$ & ns & $-4,17$ & 0 \\
\hline $1982 / 3$ & 9,73 & 2,85 & ns & 5,17 & 1,22 & ns & $-4,57$ & 0 \\
\hline $1962 / 3$ & 9,00 & 2,11 & ns & 8,00 & 4,05 & * & $-1,00$ & ns \\
\hline $1979 / 7$ & 5,07 & $-1,82$ & ns & 2,50 & $-1,45$ & ns & $-2,57$ & ns \\
\hline $1931 / 3$ & 11,17 & \begin{tabular}{|l|}
4,28 \\
\end{tabular} & * & 2,50 & $-1,45$ & ns & \begin{tabular}{|l|}
$-8,67$ \\
\end{tabular} & 000 \\
\hline $1956 / 6$ & 3,67 & $-3,22$ & ns & 1,60 & $-2,35$ & ns & $-2,07$ & ns \\
\hline $1957 / 4$ & 7,17 & 0,28 & ns & 4,00 & 0,05 & ns & $-3,17$ & ns \\
\hline $1976 / 8$ & 7,00 & 0,11 & ns & 5,00 & 1,05 & ns & $-2,00$ & ns \\
\hline $1982 / 2 A$ & 11,00 & 4,11 & * & 10,33 & 6,39 & $* *$ & $-0,67$ & ns \\
\hline $1979 / 11$ & 6,00 & $-0,89$ & ns & 1,83 & $-2,11$ & ns & $-4,17$ & 0 \\
\hline $1970 / 5$ & 6,50 & \begin{tabular}{|l|}
$-0,39$ \\
\end{tabular} & ns & 3,50 & $-0,45$ & ns & $-3,00$ & ns \\
\hline $1941 / 8$ & 9,00 & 2,11 & ns & 3,83 & $-0,11$ & ns & $-5,17$ & 00 \\
\hline $1970 / 2$ & 10,17 & 3,28 & ns & 2,93 & $-1,01$ & ns & $-7,23$ & 000 \\
\hline $1947 / 6$ & 4,67 & $-2,22$ & ns & 1,50 & $-2,45$ & ns & $-3,17$ & ns \\
\hline
\end{tabular}




\begin{tabular}{|l|l|l|l|l|l|l|l|l|}
\hline $1897 / 2$ & 10,83 & 3,95 & $*$ & 3,17 & $-0,78$ & ns & $-7,67$ & ooo \\
\hline $1941 / 9$ & 7,50 & 0,61 & ns & 5,50 & 1,55 & ns & $-2,00$ & ns \\
\hline $1982 / 1$ & 5,17 & $-1,72$ & ns & 2,33 & $-1,61$ & ns & $-2,83$ & ns \\
\hline $1970 / 1$ & 6,67 & $-0,22$ & ns & 2,83 & $-1,11$ & ns & $-3,83$ & 0 \\
\hline $1982 / 4$ & 7,83 & 0,95 & ns & 3,00 & $-0,95$ & ns & $-4,83$ & 0 \\
\hline Average (Mt & 6,89 & - & oo & 3,95 & - & & & ns \\
\hline
\end{tabular}
DL 5\%=3,80 cm;
$\mathrm{DL} 5 \%=3,76 \mathrm{~cm}$;
DL 1\%=5,05 cm;
DL $1 \%=5,03 \mathrm{~cm}$;
DL 0,1\%=6,51 cm;
DL $0,1 \%=6,64 \mathrm{~cm}$.

Examining the combined influence of the clones and the culture medium for the number of leaves / plantlets, it was observed significant positive differences (4.259 leaves and 4.926 leaves) for the 1939/2 and 1982/3 clones, on medium with PEG 2\% (Table 2), the number of leaves for these clones was not affected by drought-inducing treatments.

Table 2

Nutrition medium and clones combined influence on the number of leaves/plantlets

\begin{tabular}{|c|c|c|c|c|c|c|c|c|}
\hline \multirow[b]{2}{*}{$\begin{array}{c}\text { Clone (a)/ } \\
\text { Culture } \\
\text { medium (b) }\end{array}$} & \multicolumn{8}{|c|}{ Leaves number/ plantlets } \\
\hline & $\begin{array}{c}\text { Medium } \\
\text { MS (b }\left(b_{1}\right)\end{array}$ & $\begin{array}{l}\text { Diff. } \\
\text { (cm) }\end{array}$ & Sign. & $\begin{array}{c}\text { Medium } \\
\text { MS+PEG2\% } \\
\left(b_{2}\right)\end{array}$ & $\begin{array}{l}\text { Diff. } \\
\text { (cm) }\end{array}$ & Sign. & $\mathbf{b}_{2}-\mathbf{b}_{1}$ & Sign. \\
\hline $1939 / 4$ & 4,667 & $-3,938$ & 0 & 6,333 & $-0,741$ & ns & 1,667 & ns \\
\hline $1962 / 1$ & 9,667 & 1,062 & ns & 8,000 & 0,926 & ns & $-1,667$ & ns \\
\hline $1962 / 2$ & 8,333 & $-0,272$ & ns & 8,333 & 1,259 & ns & 0,000 & ns \\
\hline $1941 / 11$ & 7,667 & $-0,938$ & ns & 3,333 & $-3,741$ & 0 & $-4,333$ & 0 \\
\hline $1958 / 3$ & 14,000 & 5,395 & ** & 10,000 & 2,926 & ns & $-4,000$ & 0 \\
\hline $1941 / 15$ & 4,333 & $-4,272$ & 0 & 7,000 & $-0,074$ & ns & 2,667 & ns \\
\hline $1976 / 1$ & 8,000 & $-0,605$ & ns & 4,667 & $-2,407$ & ns & $-3,333$ & ns \\
\hline $1939 / 2$ & 10,333 & 1,728 & ns & 11,333 & 4,259 & * & 1,000 & ns \\
\hline $1901 / 12$ & 11,667 & 3,062 & ns & 6,333 & $-0,741$ & ns & $-5,333$ & 00 \\
\hline $1982 / 3$ & 13,000 & 4,395 & * & 12,000 & 4,926 & * & $-1,000$ & ns \\
\hline $1962 / 3$ & 10,000 & 1,395 & ns & 10,667 & 3,593 & ns & 0,667 & ns \\
\hline 1979/7 & 10,667 & 2,062 & ns & 5,333 & $-1,741$ & ns & $-5,333$ & 00 \\
\hline $1931 / 3$ & 9,333 & 0,728 & ns & 7,000 & $-0,074$ & ns & $-2,333$ & ns \\
\hline $1956 / 6$ & 6,000 & $-2,605$ & ns & 4,667 & $-2,407$ & ns & $-1,333$ & ns \\
\hline $1957 / 4$ & 6,667 & $-1,938$ & ns & 8,000 & 0,926 & ns & 1,333 & ns \\
\hline $1976 / 8$ & 7,000 & \begin{tabular}{|l|}
$-1,605$ \\
\end{tabular} & ns & 6,667 & $-0,407$ & ns & \begin{tabular}{|l|}
$-0,333$ \\
\end{tabular} & ns \\
\hline $1982 / 2 A$ & 10,000 & 1,395 & ns & 8,667 & 1,593 & ns & $-1,333$ & ns \\
\hline $1979 / 11$ & 7,333 & $-1,272$ & ns & 6,000 & $-1,074$ & ns & $-1,333$ & ns \\
\hline $1970 / 5$ & 9,667 & 1,062 & ns & 9,333 & 2,259 & ns & $-0,333$ & ns \\
\hline $1941 / 8$ & 7,000 & $-1,605$ & ns & 6,333 & $-0,741$ & ns & $-0,667$ & ns \\
\hline $1970 / 2$ & 10,000 & 1,395 & ns & 5,333 & $-1,741$ & ns & $-4,667$ & 0 \\
\hline $1947 / 6$ & 7,000 & $-1,605$ & ns & 3,667 & $-3,407$ & ns & $-3,333$ & ns \\
\hline
\end{tabular}




\begin{tabular}{|l|l|l|l|l|l|l|l|l|}
\hline $1897 / 2$ & 8,333 & $-0,272$ & ns & 5,333 & $-1,741$ & ns & $-3,000$ & ns \\
\hline $1941 / 9$ & 8,000 & $-0,605$ & ns & 7,667 & 0,593 & ns & $-0,333$ & ns \\
\hline $1982 / 1$ & 7,333 & $-1,272$ & ns & 5,667 & $-1,407$ & ns & $-1,667$ & ns \\
\hline $1970 / 1$ & 7,000 & $-1,605$ & ns & 5,667 & $-1,407$ & ns & $-1,333$ & ns \\
\hline $1982 / 4$ & 9,333 & 0,728 & ns & 7,667 & 0,593 & ns & $-1,667$ & ns \\
\hline $\begin{array}{l}\text { Mean } \\
(\mathrm{Ct})\end{array}$ & 8,605 & - & & 7,074 & - & & & \\
\hline
\end{tabular}
DL 5\%=3,721 leaves;
DL 5\%=3,820 leaves;
DL $1 \%=4,942$ leaves;
DL 1\%=5,325 leaves;
DL $0,1 \%=6,371$ leaves;
DL $0,1 \%=7,985$ leaves.

In terms of nutrient medium and clones combined influence on root length were clones that responded positively (again 1982/3) to the PEG $2 \%$ introduction and distinct very significant positive (1982/1), but also clones on which the impact was negative (1970/2) and distinct negative (1947/6) (Table 3).

Table 3

Nutrition medium and clones combined influence on root length $(\mathrm{cm})$

\begin{tabular}{|c|c|c|c|c|c|c|c|c|}
\hline \multirow{2}{*}{$\begin{array}{c}\text { Clone (a)/ } \\
\text { Culture } \\
\text { medium (b) }\end{array}$} & \multicolumn{8}{|c|}{ Root length (cm) } \\
\hline & $\begin{array}{l}\text { Medium } \\
\left.\text { MS (b } b_{1}\right)\end{array}$ & $\begin{array}{l}\text { Diff. } \\
\text { (cm) }\end{array}$ & Sign. & $\begin{array}{c}\text { Medium } \\
\text { MS+PEG2\% }\left(b_{2}\right)\end{array}$ & Diff. (cm) & Sign. & $\mathbf{b}_{2}-\mathbf{b}_{1}$ & Sign. \\
\hline $1939 / 4$ & 0,433 & $-5,640$ & 00 & 9,000 & 3,215 & ns & 8,567 & ** \\
\hline $1962 / 1$ & 5,833 & $-0,240$ & ns & 6,667 & 0,881 & ns & 0,833 & ns \\
\hline $1962 / 2$ & 3,667 & $-2,406$ & $\mathrm{~ns}$ & 7,833 & 2,048 & ns & 4,167 & * \\
\hline $1941 / 11$ & 6,667 & 0,594 & ns & 4,667 & $-1,119$ & ns & $-2,000$ & ns \\
\hline $1958 / 3$ & 6,000 & $-0,073$ & ns & 3,000 & $-2,785$ & ns & $-3,000$ & ns \\
\hline $1941 / 15$ & 0,100 & $-5,973$ & 00 & 8,267 & 2,481 & ns & 8,167 & $\star \star *$ \\
\hline $1976 / 1$ & 9,000 & 2,927 & ns & 4,967 & $-0,819$ & ns & $-4,033$ & 0 \\
\hline $1939 / 2$ & 12,333 & 6,260 & ** & 5,700 & $-0,085$ & ns & $-6,633$ & 00 \\
\hline $1901 / 12$ & 9,833 & 3,760 & * & 7,667 & 1,881 & ns & $-2,167$ & ns \\
\hline $1982 / 3$ & 9,833 & 3,760 & * & 9,500 & 3,715 & * & $-0,333$ & ns \\
\hline $1962 / 3$ & 6,333 & 0,260 & ns & 8,000 & 2,215 & ns & 1,667 & ns \\
\hline $1979 / 7$ & 3,267 & $-2,806$ & ns & 0,900 & $-4,885$ & 0 & $-2,367$ & ns \\
\hline $1931 / 3$ & 9,167 & 3,094 & $\mathrm{~ns}$ & 7,667 & 1,881 & ns & $-1,500$ & ns \\
\hline $1956 / 6$ & 5,167 & $-0,906$ & ns & 2,767 & $-3,019$ & ns & $-2,400$ & ns \\
\hline $1957 / 4$ & 7,833 & 1,760 & $\mathrm{~ns}$ & 5,167 & $-0,619$ & ns & $-2,667$ & $\mathrm{~ns}$ \\
\hline $1976 / 8$ & 6,167 & 0,094 & ns & 9,333 & 3,548 & ns & 3,167 & ns \\
\hline $1982 / 2 A$ & 7,500 & 1,427 & ns & 4,833 & $-0,952$ & ns & $-2,667$ & ns \\
\hline $1979 / 11$ & 4,667 & $-1,406$ & ns & 2,700 & $-3,085$ & ns & $-1,967$ & ns \\
\hline $1970 / 5$ & 4,167 & $-1,906$ & ns & 3,533 & $-2,252$ & ns & $-0,633$ & ns \\
\hline $1941 / 8$ & 4,667 & $-1,406$ & ns & 7,833 & 2,048 & ns & 3,167 & ns \\
\hline 1970/2 & 5,333 & $-0,740$ & ns & 1,133 & $-4,652$ & 0 & $-4,200$ & 0 \\
\hline
\end{tabular}




\begin{tabular}{|c|c|c|c|c|c|c|c|c|}
\hline $1947 / 6$ & 4,000 & $-2,073$ & ns & 0,200 & $-5,585$ & 00 & $-3,800$ & ns \\
\hline $1897 / 2$ & 7,167 & 1,094 & ns & 3,500 & $-2,285$ & ns & $-3,667$ & ns \\
\hline $1941 / 9$ & 8,500 & 2,427 & ns & 7,500 & 1,715 & ns & $-1,000$ & ns \\
\hline $1982 / 1$ & 5,667 & $-0,406$ & ns & 12,267 & 6,481 & *** & 6,600 & ** \\
\hline $1970 / 1$ & 4,000 & $-2,073$ & ns & 4,833 & $-0,952$ & ns & 0,833 & ns \\
\hline $1982 / 4$ & 6,667 & 0,594 & ns & 6,767 & 0,981 & ns & 0,100 & ns \\
\hline $\begin{array}{l}\text { Mean } \\
(\mathrm{Ct})\end{array}$ & 6,073 & - & & 5,785 & - & & & \\
\hline
\end{tabular}
DL $5 \%=3,707 \mathrm{~cm}$;
DL $5 \%=3,896 \mathrm{~cm}$;
DL $1 \%=4,924 \mathrm{~cm}$;
DL $1 \%=5,558 \mathrm{~cm}$;
DL $0,1 \%=6,347 \mathrm{~cm}$;
DL $0,1 \%=8,876 \mathrm{~cm}$.

Regarding the combined influence of nutrient medium and clones on fresh plantlets weight only 1958/3 clone responded positively to the hydric stress induced by medium MS+PEG2\%, with a significant positive difference $(0.12 \mathrm{~cm})($ Table 4$)$.

Table 4

Nutrition medium and clones combined influence on fresh plantlets weight $(\mathrm{g})$

\begin{tabular}{|c|c|c|c|c|c|c|c|c|}
\hline \multirow{2}{*}{$\begin{array}{c}\text { Clone (a)/ } \\
\text { Culture } \\
\text { medium (b) }\end{array}$} & \multicolumn{8}{|c|}{ Fresh plantlets weight $(\mathrm{g})$} \\
\hline & $\begin{array}{l}\text { Medium } \\
\left.\text { MS ( } b_{1}\right)\end{array}$ & $\begin{array}{l}\text { Diff. } \\
\text { (cm) }\end{array}$ & Sign. & $\begin{array}{c}\text { Medium } \\
\text { MS+PEG2\% }\left(b_{2}\right)\end{array}$ & $\begin{array}{l}\text { Diff. } \\
\text { (cm) }\end{array}$ & Sign. & $\mathbf{b}_{2}-\mathbf{b}_{1}$ & Sign. \\
\hline $1939 / 4$ & 0,03 & $-0,10$ & 0 & 0,04 & $-0,06$ & ns & 0,01 & ns \\
\hline $1962 / 1$ & 0,07 & $-0,06$ & ns & 0,14 & 0,05 & ns & 0,07 & ns \\
\hline $1962 / 2$ & 0,05 & $-0,08$ & ns & 0,12 & 0,02 & ns & 0,07 & ns \\
\hline $1941 / 11$ & 0,17 & 0,05 & ns & 0,02 & $-0,07$ & ns & $-0,15$ & 00 \\
\hline $1958 / 3$ & 0,29 & 0,17 & 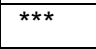 & 0,22 & 0,12 & ${ }^{*}$ & $-0,07$ & ns \\
\hline $1941 / 15$ & 0,02 & $-0,11$ & 0 & 0,08 & $-0,01$ & ns & 0,06 & ns \\
\hline $1976 / 1$ & 0,14 & 0,01 & ns & 0,13 & 0,03 & ns & $-0,01$ & ns \\
\hline $1939 / 2$ & 0,15 & 0,02 & ns & 0,11 & 0,01 & ns & $-0,04$ & ns \\
\hline $1901 / 12$ & 0,21 & 0,08 & ns & 0,06 & $-0,04$ & ns & $-0,15$ & 00 \\
\hline $1982 / 3$ & 0,12 & 0,00 & ns & 0,10 & 0,00 & ns & $-0,03$ & ns \\
\hline $1962 / 3$ & 0,11 & $-0,01$ & ns & 0,14 & 0,04 & ns & 0,03 & ns \\
\hline $1979 / 7$ & 0,17 & 0,04 & $\mathrm{~ns}$ & 0,07 & $-0,02$ & ns & $-0,10$ & 0 \\
\hline $1931 / 3$ & 0,18 & 0,05 & ns & 0,12 & 0,02 & $\mathrm{~ns}$ & $-0,06$ & $\mathrm{~ns}$ \\
\hline $1956 / 6$ & 0,08 & $-0,05$ & ns & 0,05 & $-0,04$ & ns & $-0,02$ & ns \\
\hline $1957 / 4$ & 0,14 & 0,01 & ns & 0,07 & $-0,02$ & ns & $-0,07$ & $\mathrm{~ns}$ \\
\hline $1976 / 8$ & 0,14 & 0,01 & ns & 0,11 & 0,02 & ns & $-0,03$ & ns \\
\hline $1982 / 2 A$ & 0,14 & 0,02 & ns & 0,17 & 0,07 & ns & 0,03 & $\mathrm{~ns}$ \\
\hline $1979 / 11$ & 0,11 & $-0,02$ & ns & 0,10 & 0,00 & ns & $-0,01$ & ns \\
\hline $1970 / 5$ & 0,11 & $-0,02$ & ns & 0,08 & $-0,02$ & ns & $-0,03$ & $\mathrm{~ns}$ \\
\hline $1941 / 8$ & 0,15 & 0,02 & ns & 0,09 & $-0,01$ & ns & $-0,06$ & ns \\
\hline
\end{tabular}




\begin{tabular}{|l|l|l|l|l|l|l|l|l|}
\hline $1970 / 2$ & 0,17 & 0,04 & ns & 0,07 & $-0,03$ & ns & $-0,10$ & o \\
\hline $1947 / 6$ & 0,09 & $-0,04$ & ns & 0,03 & $-0,06$ & ns & $-0,05$ & ns \\
\hline $1897 / 2$ & 0,16 & 0,03 & ns & 0,07 & $-0,03$ & ns & $-0,09$ & ns \\
\hline $1941 / 9$ & 0,11 & $-0,01$ & ns & 0,11 & 0,01 & ns & $-0,01$ & ns \\
\hline $1982 / 1$ & 0,10 & $-0,03$ & ns & 0,09 & 0,00 & ns & 0,00 & ns \\
\hline $1970 / 1$ & 0,09 & $-0,04$ & ns & 0,12 & 0,02 & ns & 0,03 & ns \\
\hline $1982 / 4$ & 0,16 & 0,03 & ns & 0,11 & 0,01 & ns & $-0,05$ & ns \\
\hline $\begin{array}{l}\text { Mean } \\
\text { (Ct) }\end{array}$ & 0,13 & - & & 0,10 & - & & & \\
\hline
\end{tabular}
DL 5\%=0,093 g;
DL 5\%=0,092 g;
DL $1 \%=0,124 \mathrm{~g}$;
DL $1 \%=0,122 \mathrm{~g} ; \mathrm{v}$
DL $0,1 \%=0,160 \mathrm{~g}$.
DL $0,1 \%=0,158 \mathrm{~g}$

In vitro simulation of water stress conditions can lead to an efficient system of differentiation of potato clones in terms of determining root growth, leaf number and other parameters, so that the identification of drought tolerant and / or sensitive genotypes can be accomplished in a shorter time.

\section{CONCLUSIONS}

One of the great challenges of the next decade is to reduce any effect of climate change on production, paying more attention to maintaining yield under thermo-hydric stress.

In case of medium with PEG $2 \%$ concentration 1982/3 clone was identified with high values (12 leaves and $9.5 \mathrm{~cm}$ ) and significant positive differences $(4.926$ leaves and $3.715 \mathrm{~cm}$ ), for the number of leaves/plantlets and for root length. Also, $1939 / 2$ was identified with high values for the number of leaves/plantlets (11.333 leaves, with significant positive difference 4.259 leaves) and 1982/1 clone for root length $(12.267 \mathrm{~cm}$, with very significant positive difference $6.481 \mathrm{~cm})$. At the same time, 1958/3 clone presented high values for two of the analyzed parameters: plantlet length $(12.17 \mathrm{~cm})$ and fresh plantlets weight $(0.22 \mathrm{~g})$, when was applied PEG $2 \%$ in the nutrition medium, with very significant positive $(8.22 \mathrm{~cm})$ and significant positive $(0.12 \mathrm{~g})$ differences.

In order to make recommendations regarding the cultivation areas and what varieties are suitable for each of them, the pedoclimatic conditions must be taken into account, as well as the crop yield, because during the vegetation may occur periods with humidity deficit.

\section{ACKNOWLEDGMENT}

The paper is part of the Project ADER 4.1.1. "Obtaining new potato varieties with superior ability to respond to abiotic and biotic stress by using the genetic determinism of tolerance traits" funded by Ministry of Agriculture and Rural Development.

\section{REFERENCES}

Ahmad R., Waraich E.A., Ashra M.Y., Ahma S., Aziz T., 2013. Does nitrogen fertilization enhance drought tolerance in sunflower? A review. Journal of Plant Nutrition 37: 942-963. 
Albiski F., Najla S., Sanoubar R., Alkabani Nour, Murshed R.2012. In vitro screening of potato lines for drought tolerance. Physiol Mol Biol Plants.DOI 10.1007/s12298-012-0127-5.

Baciu Anca, 2013. Reaction of native potato varieties to water stress. Journal of Horticulture, Forestry and Biotechnology, vol. 17(2), 80- 86.

Cabello R., Mendiburu F., Bonierbale Merideth, Monneveux P., Roc, W., Chujoy, E. 2012. Large-Scale Evaluation of Potato Improved Varieties, Genetic Stocks and Landraces for Drought Tolerance. 2012. American Journal of Potato Research 89 (5), 400-410.

Evers D., Lefevre I., Legay S., Lamoureux D., Hausman J.F., Rosales R.O.G. 2010. Identification of drought-responsive compounds in potato through a combined transcriptomic and targeted metabolite approach. J. Exp. Bot. 61, 2327-2343. 10.1093/jxb/erq060.

Deblonde P.M.K., Haverkort, A.J., Ledent J.F. 1999. Responses of early and late potato cultivars to moderate drought conditions: agronomic parameters and carbon isotope discrimination. Eur J Agron 11:91-105.

Gopal J., Iwama K. 2007. In vitro screening of potato against water-stress mediated through sorbitol and polyethylene glycol; Plant Cell Rep, 26:693-700.

Hassan N.S., Shaaban L.D., El-Sayed A.H., Seleem E.E. 2004. In Vitro selection for water stress tolerant callus line of Helianthus annus L Cv Myak. Int $\mathrm{J}$ Agric Biol 6, 1-13.

Hussain M., Malik M.A., Farooq M., Ashraf M.Y., Cheema M.A. 2008. Improving drought tolerance by exogenous application of glycine-betaine and salicylic acid in sun flower. J Agron Crop Sci 194:193 -199.

Jefferies R.A., MacKerron D.K.L. 1987. Aspects of the physiological basis of cultivar differences in yield of potato under droughted and irrigated conditions. Potato Res 30: $201-217$.

Lipavska, H., Vreugdenhil, D., 1996. Uptake of mannitol from the media by in vitro grown plants, Plant Cell Tissue Org. Cult. 45 (1996) 103-107.

Manoj K., Uday D. 2007. Gradient in vitro testing of tomato (Solanum lycopersicum) genotypes by inducing water deficit: a new approach to screen germplasm for drought tolerance, Asian J. Plant Sci. 6:934-940.

Monneveux, P., Ramírez, D.A., Pino, M.T., 2013. Drought tolerance in potato (S. tuberosum L.): Can we learn from drought tolerance research in cereals? Plant Sci. 205-206:76-86. doi: 10.1016/j.plantsci.2013.01.011.

Monneveux P., Ramirez D.A., Khan M.A., Raymundo R.M., Loayza H., Quiroz R. 2014. Drought and heat tolerance evaluation in potato (Solanum tuberosum L.). Potato Research. (Netherlands). ISSN 0014-3065. 57(3-4), 225-247.

Morar G. 1999. Cultura cartofului. Risoprint ed., Cluj Napoca.

Murashige T., Skoog F. 1962. A revised medium for rapid growth and bioassays with tobacco tissue cultures. Physiologia Plantarum,15, 473-497.

Obidiegwu J.E., Bryan G.J., Jones H.G., Prashar A. 2015. Coping with drought: stress and adaptive responses in potato and perspectives for improvement. Frontiers in plant science 6:542 DOI: 10.3389/fpls.2015.00542.

Yaniv Z., Werker E. 1983. Absorption and secretion of polyethylene glycol by Solanaceous plants, J. Exp. Bot. 34, 1577-1584. 